\title{
Elevated systemic interleukin-7 in patients with colorectal cancer and individuals at high risk of cancer: association with lymph node involvement and tumor location in the right colon
}

\author{
Malgorzata Krzystek-Korpacka ${ }^{1}\left(\mathbb{1} \cdot\right.$ Marek Zawadzki $^{2} \cdot$ Katarzyna Neubauer $^{3} \cdot$ \\ Iwona Bednarz-Misa $^{1} \cdot$ Sabina Górska $^{4} \cdot$ Jerzy Wiśniewski $^{1} \cdot$ Wojciech Witkiewicz $^{2,5}$. \\ Andrzej Gamian ${ }^{1,4}$
}

Received: 24 April 2016 / Accepted: 14 November 2016 / Published online: 19 November 2016

(C) The Author(s) 2016. This article is published with open access at Springerlink.com

\begin{abstract}
Interleukin (IL)-7 is a cytokine essential for protective immunity, and it is considered as a promising agent for cancer immunotherapy. Recent studies, however, appear to associate IL-7 with aggressiveness of solid tumors. The IL-7 has been less studied in colorectal cancer (CRC) and conditions associated with increased risk of CRC development. To explore IL-7 status in bowel diseases, it was measured immunofluorometrically in 431 individuals (110 with CRC) by using Luminex platform. A level of IL-7 in CRC patients was significantly higher than in controls, did not differ from those with adenomas, but was lower than in both active and inactive inflammatory bowel disease (IBD) cases. In CRC, IL-7 was higher in patients with lymph node and distant metastases and with tumors located in right colon. In adenomas, IL-7 elevation was associated exclusively with villous growth pattern, while in IBD, circulating IL-7 reflected clinical activity of Crohn's disease and ulcerative colitis. Systemic TNF $\alpha$, IL-10, and PDGF-BB were independent predictors of circulating IL-7. In summary,
\end{abstract}

Malgorzata Krzystek-Korpacka

gosia.krzystek@gmail.com;

malgorzata.krzystek-korpacka@umed.wroc.pl

1 Department of Medical Biochemistry, Wroclaw Medical University, ul. Chalubinskiego 10, 50-368 Wroclaw, Poland

2 Department of Surgical Oncology, Regional Specialist Hospital, Wroclaw, Poland

3 Department of Gastroenterology and Hepatology, Wroclaw Medical University, Wroclaw, Poland

4 Laboratory of Medical Microbiology, Ludwik Hirszfeld Institute of Immunology and Experimental Therapy, Polish Academy of Sciences, Wroclaw, Poland

5 Research and Development Centre at Regional Specialist Hospital, Wroclaw, Poland

our study is the first to demonstrate IL-7 elevation in CRC in association with metastatic disease and tumor location. Both associations should be considered when designing IL7-based immunotherapies for CRC. Further studies on IL-7 functionality in CRC are necessary.

Keywords Interleukin-7 - Colorectal cancer . Inflammatory bowel disease $\cdot$ Adenomas $\cdot$ Lymph node metastasis · Immunity
Abbreviations
ASA classification
American Society of Anes- thesiologists classification of patients' physical status
CD
CDAI
CRC
DSM IV
FGF
IBD
ICD-10
IP10
MCP
MDAI
NINCDS-ADRDA
PDGF
Crohn's disease
Crohn's disease activity index
Colorectal cancer
Diagnostic and statistical manual of mental disorders Fibroblast growth factor Inflammatory bowel disease International statistical classi- fication of diseases and related health problems Interferon gamma-induced protein 10
Monocyte chemoattractant protein
Mayo disease activity index National Institute of Neurolog- ical and Communicative Dis- orders and Stroke-Alzheimer's Disease and Related Disorders Association Platelet-derived growth factor 
$\mathrm{UC}$

Ulcerative colitis

UICC TNM classification

Union Internationale Contre le Cancer tumor-node-metastasis system for classification of malignant tumors

VEGF Vascular endothelial growth factor

\section{Introduction}

Colorectal cancer (CRC) remains one of the commonest and most lethal cancers worldwide [1, 2]. CRC development is frequently preceded by formation of adenomas. Although benign, these lesions may develop into cancer through a sequence of genetic and epigenetic alterations. A prevalence of the adenomas in older population is relatively high $[3,4]$. Inflammatory bowel disease (IBD) is another pathology closely associated with an increased risk of CRC development. Inflammatory character of the disease combined with its incurableness, chronicity, and relapsingremitting nature eventually causes dysplasia and facilitates neoplastic transformation [5]. While survival rates for CRC patients with the resectable tumors are improving, the prognosis for patients with advanced CRC remains poor, rendering development of new therapeutic strategies a necessity. Immunotherapy, an approach based on boosting immune system to fight cancer, is a promising strategy that may improve outcomes for the CRC patients with metastatic disease or cancers resistant to chemo- and/or radiotherapy. It has also potential to prevent disease relapse, following radical tumor resection or eradication, facilitated by residual circulating cells, micrometastases or cancer stem cells [6].

Interleukin (IL)-7 is a pleiotropic cytokine, crucial for development and homeostasis of lymphocytes $\mathrm{T}$, acting as their mitogen, growth and survival factor. IL-7 is ubiquitous and mainly tissue-derived cytokine [7-9]. Owing to its central role in innate and adaptive immunity, IL-7 has been listed as one of the "Top Agents with High Potential for Use in Treating Cancer" by the panel of experts at the National Cancer Institute Immunotherapy Agent Workshop in 2007 [10]. IL-7 may up-regulate tumor-directed immune responses in a number of ways, including, but not limited to, enhancement of the cellular $\left(\mathrm{T}_{\mathrm{h}} 1\right)$ immune response or selective expansion of the tumor-redirected cytotoxic $\mathrm{T}$ lymphocytes $[8,9,11,12]$. Unlike the other $\gamma$-chain cytokines tested as therapeutics (e.g. IL-2), application of IL-7 in cancer immunotherapy is particularly appealing, because it does not induce hyperinflammation [13, 14]. Since IL-7 plays a role in both predisposing to autoimmunity and in perpetuating autoimmune inflammation [7], targeting IL-7/IL7R signaling appears to be an attractive therapeutic option for preventing CRC [15].

However, there is an increasing number of reports showing IL-7 to be overexpressed by solid tumors [11, 16-19] and being elevated in sera of the cancer patients [20-24]. Although functional data are still scanty, available evidence seems to link IL-7 overexpression with tumor aggressiveness, metastasis, and unfavorable prognosis [11, 19].

In the light of growing interest in immunotherapy based on this cytokine and controversies associated with its expression by solid tumors, we aimed at exploring IL-7 status in CRC and conditions linked to an increased risk of its development, identifying factors contributing to this cytokine elevation.

\section{Materials and methods}

\section{Study population}

Systemic IL-7 was measured in serum samples from 431 individuals: 110 with CRC, 21 with adenomas, 171 with IBD (133 with active and 38 with inactive disease; 97 with Crohn's disease (CD) and 74 with ulcerative colitis (UC)), and 129 controls. Enrolled CRC patients were admitted to the Department of Surgical Oncology, Regional Specialist Hospital in Wroclaw in years 2013-2015 for curative resection of histologically confirmed adenocarcinoma of colon or rectum. Patients aged $<18$ years, with poor overall physical status (ASA physical status classification system $>3$ ), requiring emergency surgery, with gross metastatic disease or locally advanced cancers not amenable to curative resection were excluded. Resected tumors were staged pathomorphologically according to UICC TNM 7th edition from 2010 and stage distribution is given in Table 1. Individuals with IBD and adenomas were inpatients of the Department of Gastroenterology and Hepatology of Wroclaw Medical University. Patients with indeterminate colitis or the co-existence of other severe systemic diseases, malignancies, liver diseases, or pregnancies were excluded. Crohn's Disease Activity Index (CDAI) was applied for the assessment of CD activity and the Mayo Scoring System (MDAI) for UC. IBD patients, with few exceptions, were treated with $5^{\prime}$-aminosalicylate derivatives. Twelve patients with adenomas had multiple polyps, in nine the polyps were larger than $10 \mathrm{~mm}$, and in eight the polyps had villous growth pattern. Control group consisted of healthy volunteers from hospital staff, outpatients of Research, Science, and Educational Center of Dementia Diseases, Scinawa, Poland, suffering from headaches or mild cognitive disorders (Alzheimer disease and other forms of dementia were excluded using neuroimaging and the following criteria: 
Table 1 Circulating IL-7 and CRC advancement

\begin{tabular}{|c|c|c|c|}
\hline & $N$ & Mean IL-7 (95\% CI) & $P$ \\
\hline \multicolumn{3}{|c|}{ Disease stage (UICC TNM7th) } & 0.027 \\
\hline 0 & 5 & $5.95(4-8.9)$ & \\
\hline I & 8 & $8.32(5.9-11.9)$ & \\
\hline II & 41 & $10.59(8.2-13.7)$ & \\
\hline III & 47 & $12.74(10.7-15.1)$ & \\
\hline IV & 9 & $19.63(7.2-80.7)$ & \\
\hline \multicolumn{3}{|c|}{ Primary tumor $(T)$} & 0.162 \\
\hline Tis & 5 & $5.95(4-8.9)$ & \\
\hline $\mathrm{T} 1$ & 1 & 7.45 & \\
\hline $\mathrm{T} 2$ & 11 & $10.73(7.2-16)$ & \\
\hline $\mathrm{T} 3$ & 62 & $11.19(9.2-13.6)$ & \\
\hline $\mathrm{T} 4$ & 31 & $14.22(10.6-19.1)$ & \\
\hline \multicolumn{3}{|c|}{ Lymph node involvement $(N)$} & 0.018 \\
\hline No (N0) & 54 & $9.69(7.9-11.9)$ & \\
\hline Yes (N1/N2) & $56(33 / 23)$ & $13.66(11.2-16.7)$ & \\
\hline \multicolumn{3}{|c|}{ Distant metastases $(M)$} & 0.030 \\
\hline M0 & 101 & $11.01(9.6-12.6)$ & \\
\hline M1 & 9 & $19.63(72-53.6)$ & \\
\hline \multicolumn{3}{|c|}{ Grade of differentiation $(G)$} & 0.693 \\
\hline G1 & 9 & $9.35(4.4-19.8)$ & \\
\hline $\mathrm{G} 2$ & 79 & $12.11(10.1-14.5)$ & \\
\hline G3 & 15 & $12.94(10.5-16)$ & \\
\hline G4 & 1 & 7.45 & \\
\hline
\end{tabular}

ICD-10, DSM IV, and NINCDS-ADRDA as revised in 2007), patients with non-malignant and non-inflammatory bowel diseases (irritable bowel syndrome, diverticulosis, hemorrhoids) and blood donors, whose sera were kindly provided by Regional Center of Blood Donation and Therapeutics in Wroclaw, Poland. Inclusion criteria for control group were: age $>18$ years, overall good health condition, and willingness to participate. Exclusion criteria were pregnancy, active inflammation, known severe systemic disease, dementia or depression.

Subjects distribution based on gender (females/males) in CRC, adenoma, active IBD, inactive IBD and control cohorts did not differ significantly $(p=0.673)$ and was as follows: $43 / 67,11 / 10,63 / 70 ; 16 / 22$, and 57/72. Age distribution in CRC, adenoma, active IBD, inactive IBD, and in control cohorts was as follows: 65 years (95\% CI 64-68), 61.5 years (56-77), 33 years (31-36), 40 years (29-44), and 50 years (42-56). Due to natural history of the diseases, median age in both IBD cohorts differed significantly from other groups $(p<0.05)$. However, IL-7 did not correlate with age in any evaluated cohort: $p=0.174$ for CRC, $p=0.959$ for adenomas, $p=0.156$ for active IBD, $p=0.681$ for inactive IBD, and $p=0.196$ for controls. Nevertheless, control group was divided into subgroups based on age: $<45$ years (median age 34 years
(33-38), $n=62$ ) to match age distribution in IBD patients $(p=0.579)$ and $\geq 45$ years (median age 64 years $(62-65)$, $n=67)$ to match age distribution in CRC and adenoma patients $(p=0.295)$.

The study protocol was approved by the Medical Ethics Committees of Wroclaw Medical University and of Regional Specialist Hospital, and the study was conducted in accordance with the Helsinki Declaration of 1975, as revised in 1983, and an informed consent has been obtained from all patients.

\section{Analytical methods}

Blood was drawn in a fasting state prior to any procedure by venipuncture, clotted for $30 \mathrm{~min}$, and subsequently centrifuged $(15 \mathrm{~min}, 720 \times \mathrm{g})$. Collected serum was aliquoted and kept frozen at $-80^{\circ}$ until examination. Samples were measured in duplicates or triplicates by means of flow cytometry-based method utilizing magnetic microspheres conjugated with monoclonal antibodies using the BioPlex 200 platform with HRF (Bio-Rad, USA), incorporating Luminex $\mathrm{xMAP}^{\circledR}$ technology. Bio-Plex Pro $^{\mathrm{TM}}$ Human Cytokine, Chemokine, and Growth Factor Magnetic BeadBased Assays were used according to manufacturer's instructions to measure the levels of IL-7 and IL- $1 \beta$, IL-6, IL-8, IL-10, IL-12(p70), IFN $\gamma$, MCP-1, MIP-1 $\alpha$, MIP-1 $\beta$, IP-10, FGF2, PDGF-BB, TNF $\alpha$, and VEGF-A. Standard curves were drawn using 5-PL logistic regression, and the data were analyzed using BioPlex Manager 6.0 software.

\section{Statistical analysis}

Chi-squared test was applied to assess the normality of data distribution. Homogeneity of variation was evaluated using Levene test. Data were log-transformed to obtain normality. Normally distributed data are presented as geometric means and analyzed using $t$ test for independent samples with Welch correction if appropriate or one-way ANOVA with Bonferroni correction for multiple testing and Student-Newman-Keuls post hoc test. Non-normally distributed data are presented as medians and analyzed using Kruskal-Wallis $H$ test. Both geometric means and medians are accompanied by $95 \%$ CI. Two-way ANOVA was employed to co-exam the effects of tumor location and regional metastases on IL-7. Correlation analysis was conducted using Spearman rank test $(\rho)$ or Pearson test $(r)$, depending on data character and distribution. Frequency analysis was conducted using Chi-square test. Enter and stepwise method of multivariate analysis was used to discern independent predictors of $\mathrm{IL}-7$ and to determine partial correlation coefficients (net correlation with the effects of other variables removed). To limit the number of variables, we used two-step procedure. It allowed us to 
Fig. 1 Systemic IL-7 in colorectal cancer and cancer high-risk conditions. a Analysis on whole study population; b Analysis limited to IBD patients and their age-matched controls; c Analysis limited to CRC and adenoma patients and their age-matched controls. ADN, adenomas; CONT, controls; CRC, colorectal cancer; IBDa, active inflammatory bowel disease; IBDn, inactive inflammatory bowel disease. Data presented as medians with 95\% CI and analyzed using Kruskal-Wallis $H$ test. Lower script letters indicate significant between-group differences. Outlying observations are presented together as open circles above dashed line and accompanied by IL-7 values

eliminate insignificant variables in a first step so the ratio of variables-to-cases in a final model was acceptable $(1: 16)$. The following criteria were used: $p<0.05$ for entering and $p>0.1$ for removing of variables. Coefficient of determination adjusted for the number of independent variables in the regression model ( $R^{2}$-adjusted) represents the goodness of fit of the model. All calculated probabilities were twotailed, and $p$ values $\leq 0.05$ were considered statistically significant. The statistical analysis was conducted using MedCalc Statistical Software version 16.2.0 (MedCalc Software bvba, Ostend, Belgium; https://www.medcalc.org; 2016).

\section{Results}

\section{Circulating IL-7 in bowel diseases}

The concentration of IL-7 determined in sera from CRC patients was significantly higher than in controls, did not differ from patients with adenomas, and was significantly lower than in IBD, in both active and inactive cases of this disease (Fig. 1a).

Patients with adenomas had higher IL-7 than controls and lower than patients with active IBD, whose cytokine levels were significantly more elevated than in any other group except for inactive disease.

Owing to natural history of IBD and CRC with the diseases onset, respectively, early and late in life, the control group could not be age-matched to both CRC and IBD cohorts. Although systemic IL-7 did not correlate with age, we re-analyzed the data after dividing our controls into two subgroups: one including younger individuals (age $<45$ years) to be compared with IBD patients and the other consisting of older individuals (age $\geq 45$ years) to be compared with adenoma and CRC patients. It allowed us to confirm significance of the elevation of IL-7 in CRC, adenoma, and IBD patients as compared to controls in age-matched analysis (Fig. 1b, c).

\section{Circulating IL-7 and CRC advancement}

A relation between CRC advancement and serum concentration of IL-7 is summarized in Table 1. Cytokine
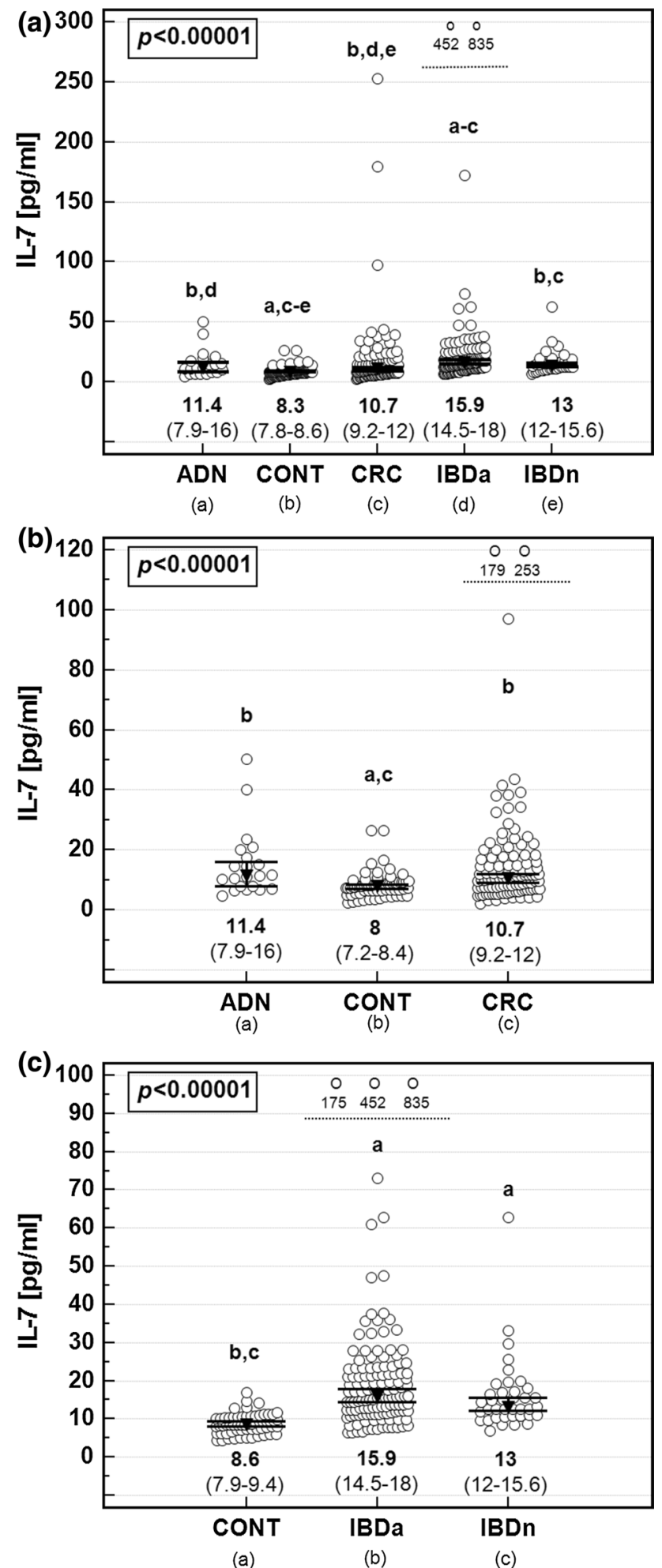

levels increased along with the disease stage $(\rho=0.29$, $p=0.002$ ) with a significant difference between stages 0 and IV. Significant elevation in IL-7 concentration was associated also with lymph node involvement and presence of distant metastases. There was a weak positive 
correlation between IL-7 and T stage ( $\rho=0.22, p=0.021)$ and between IL-7 and a number of metastatic lymph nodes $(\rho=0.28, p=0.013, n=77)$.

\section{Circulating IL-7 and tumor location}

Circulating IL-7 also differed with respect to tumor location. Its concentration was significantly higher in serum of the patients with tumors localized in right colon (Fig. 2).

There was no difference in stage distribution ( $p=0.493)$, lymph node involvement $(p=0.811)$, or presence of distant metastases $(p=0.265)$ between rightsided CRC and other cancer locations. Moreover, when coexamined in two-way ANOVA, both lymph node involvement $(F=5.29, p=0.023)$ and tumor location $(F=6.35$, $p=0.013)$ were significantly and independently from each other associated with IL-7.

\section{Circulating IL-7 and CRC high-risk conditions}

There were no differences in systemic IL-7 between CD and UC, regardless whether patients with active or nonactive disease were compared. Cytokine levels positively correlated with the indices of clinical activity of both Crohn's disease-CDAI $(\rho=0.3, p=0.007)$ and ulcerative colitis-Mayo score $(\rho=0.3, p=0.008)$.

In adenoma group, there was no difference in IL-7 with respect to number of polyps (one $v s$. multiple, $p=0.983$ ) or their size $(10<\mathrm{vs.} \geq 10 \mathrm{~mm}, p=0.993)$. However, IL-7 was significantly higher in patients with adenomas displaying a villous growth pattern (villous adenomas and tubulovillous adenomas) as compared to tubular adenomas: $20.6 \mathrm{pg} / \mathrm{ml}$ (95\% CI 12.9-32.7) and 9.2 pg/ml (7.2-11.8), $p=0.001$. Cytokine levels in patients with tubular adenomas did not differ significantly from controls $(p=0.182)$.

\section{Correlation pattern of IL-7 with other cytokines}

We evaluated the association between IL-7 and other cytokines and growth factors selected from among pro-/ anti-inflammatory and Th1/Th2 cytokines (IL-1 $\beta$, IL-6, TNF $\alpha$, IFN $\gamma$, IL12p(70), and IL-10), chemokines (MCP-1, MIP- $1 \alpha$, MIP- $1 \beta$, and IL- 8 ), and pro- and anti-angiogenic factors (IP10, FGF2, PDGF-BB, and VEGF-A). Circulating IL-7 was correlated with most of the evaluated cytokines and growth factors, except for MIP-1 $\beta$ and IP10. Particularly strong correlations were observed for TNF $\alpha$ and IFN $\gamma$ (Table 2).

Statistically, the examined cytokines were intertwined and to discern their independent associations and determine partial correlation coefficients, we applied two-step multiple regression. First, we entered all cytokines significantly correlated with IL-7 in univariate analysis as independent

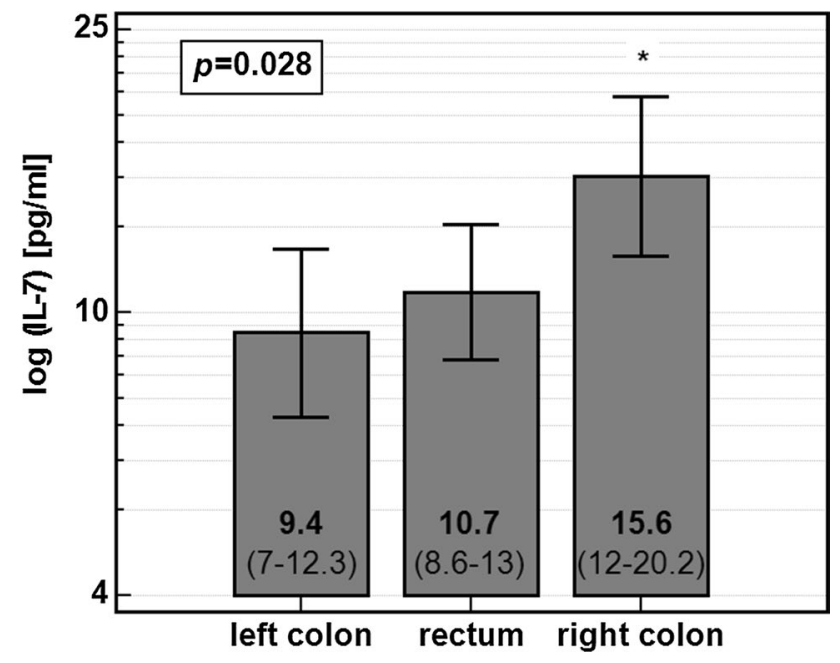

Fig. 2 Circulating IL-7 and tumor location. Data presented as geometric means with 95\% CI and analyzed using one-way ANOVA with Bonferroni corrections for multiple testing. Asterisk significantly different form other groups

variables. Then, cytokines with $\mathrm{p}>0.1$ were removed and remaining cytokines re-entered to analysis and stepwise method of model building was used. TNF $\alpha$, IL-10, IL-1 $\beta$, MCP-1, and PDGF-BB were entered in final model and $\mathrm{TNF} \alpha$, IL-10, and PDGF-BB remained as independent predictors of IL-7 explaining $64 \%$ of its variability (Table 3 ).

\section{Discussion}

In the context of cancer disease, interleukin (IL)-7 is viewed as a key therapeutic factor, while substantially less attention has been devoted to its possible association with the disease development. Occupying central position in innate and adaptive immunity, IL-7 with its repertoire of antibacterial, anti-fungal, anti-viral, and anti-tumor activities is considered as "a critical enhancer of protective immunity" [25]. At the same time, mitogenic activity of IL-7 toward lymphoid populations might result in lymphoproliferative diseases. Accordingly, the cytokine has been implicated in the development and progression of leukemias and lymphomas [11, 17]. IL-7 association with solid tumors is relatively new and unexplored aspect of the research. Nevertheless, there is a growing body of evidence linking IL-7/IL-7R signaling with tumor aggressiveness, metastatic disease, and unfavorable prognosis [11, 17, 19, 26]. There is paucity of data concerning IL-7 in CRC and conditions predisposing to the disease. IL-7 expression was found in tumor biopsies obtained from CRC patients [16], and it was reported by Crucitti et al. [21] to be significantly elevated in sera, while others linked IL-7 elevation to metastatic disease [20] and poorer survival [24]. Our findings 
Table 2 IL-7 correlation pattern with inflammatory, Th1/Th2, chemotactic, and angiogenic cytokines

\begin{tabular}{lll}
\hline & Cytokine & Correlation coefficient, $p$ \\
\hline Inflammatory, Th1/Th2 & IL-1 $\beta$ & $0.52, p<0.0001$ \\
& TNF $\alpha$ & $0.65, p<0.0001$ \\
& IL-6 & $0.40, p<0.0001$ \\
& IL-12(p70) & $0.42, p<0.0001$ \\
& IFN $\gamma$ & $0.69, p<0.0001$ \\
& IL-10 & $0.57, p<0.0001$ \\
Chemotactic & MCP1 & $0.26, p=0.007$ \\
& MIP1 $\alpha$ & $0.34, p<0.001$ \\
& IL-8 & $0.21, p=0.030$ \\
& MIP1 $\beta$ & $0.17, p=0.072$ \\
Angiogenic & FGF2 & $0.53, p<0.0001$ \\
& PDGF-BB & $0.54, p<0.0001$ \\
& VEGF-A & $0.20, p=0.041$ \\
& IP10 & $0.13, p=0.176$ \\
\hline
\end{tabular}

Table 3 Multiple regression analysis with IL-7 as dependent variable

\begin{tabular}{llll}
\hline & Partial correlation coefficient & statistics $t$ & $p$ \\
\hline IL-10 & 0.494 & 5.78 & $<0.0001$ \\
PDGF-BB & 0.411 & 4.63 & 0.002 \\
$\mathrm{TNF} \alpha$ & 0.582 & 7.36 & $<0.0001$ \\
$R^{2}$ adjusted & 0.642 & & \\
$F$ ratio, $p$ & $66.1, p<0.0001$ & & \\
\hline
\end{tabular}

confirm systemic elevation of IL-7 in CRC and expand the knowledge by placing it in the broad context of health and non-malignant bowel diseases associated with increased risk of CRC development.

Contrary to data presented by Henry et al. [27] but corroborating findings of Comstock et al. [28], we have observed that circulating IL-7 was elevated in patients with adenomas, as compared to controls. Similar increase in several other cytokines was reported elsewhere [28-31]. To resolve discrepancy between the reports, we investigated IL-7 in the context of adenomas, their number, size, and growth pattern. Detailed analysis revealed that IL-7 elevation coincided exclusively with villous growth pattern, while systemic IL-7 in patients with tubular adenomas was similar to controls. The dominant villous growth pattern is associated with large adenomas considered "advanced," and they are more likely to progress into carcinoma [5]. The significance of IL-7 elevation in advanced adenomas needs further clarification, but it might be of interest to mention that in benign prostatic hyperplasia IL-7 has been shown to stimulate proliferation of the prostatic cells (reviewed in [17]).

Chronic inflammation-induced dysplasia is another risk factor for neoplastic transformation, and the odds of CRC development are six-times higher in IBD patients than in general population. Moreover, IBD patients are more likely to have multiple synchronous CRC and their mortality is higher than in a case of sporadic cancers [32]. Corroborating reports published by others [33-35], we found IL-7 to be elevated in IBD as compared to controls. IL-7 elevation was more pronounced than in $\mathrm{CRC}$, regardless whether the disease was active or IBD patients were in remission. Still, IL-7 weakly corresponded with clinical activity of both Crohn's disease and ulcerative colitis. IL-7 elevation observed in IBD patients is consistent with findings by others who reported higher percentage of activated and cycling CD4+ and CD8+ T cells and T cells expressing IFN $\gamma$ as well as their increased resistance to apoptosis as compared to healthy individuals $[15,36]$. The up-regulation of IL-7 signaling in IBD had a worse treatment-refractory disease course, probably due to enriched pool of antigen-specific memory $\mathrm{T}$ cells which, upon future re-encounter with antigen, would facilitate faster and more potent response from CD8 effector cells [37]. Intuitively, elevated IL-7 in IBD might confer at least some degree of protection against $\mathrm{CRC}$ development by promoting $\mathrm{T}$ cell mediated antitumor responses. However, $\mathrm{T}$ cell responsiveness to IL-7 has recently been demonstrated to be impeded by their exposure to inflammatory cytokines [38].

IL-7 is produced by stromal and epithelial cells as well as endothelial and immune cells. Its serum level is mainly regulated through the up-take by lymphocytes, thus elevating IL-7 in conditions associated with lymphopenia. The relevance of cytokine serum elevation found in cancer or the role of IL-7 expression and secretion by tumor cells remains elusive. Nonetheless, accumulated data seem to point at this cytokine involvement in metastasis $[9,11,19]$. Accordingly, our analysis of IL-7 association with clinicopathological features of CRC revealed that cytokine levels were more elevated in patients with lymph node involvement. Moreover, IL-7 levels were dependent on PDGFBB and correlated with FGF2, VEGF-A, and IL-8, other potent angiogenic growth factors. Similar to Berghella's et al. [20], we observed an increase in IL-7 in CRC patients with distant metastases despite a limited number of stage IV CRCs in current cohort. Our observations are consistent with lymphangiogenic role attributed to IL-7 [17, 39]. In lung cancer, IL-7 protein expression has correlated with increased lymphovascular density, lymph node metastases, advanced clinical stage, VEGF-D, and cyclin D expression [39] and has been identified as a potent marker for bone metastasis [19]. In fact, bone invasion has been required to trigger an intense cytokine production and its increase in sera [19]. Similar association with nodal involvement, tumor aggressiveness and worse prognosis was observed for IL-7 and expression of its receptor by Al-Rawi et al. [40] in breast cancer. Moreover, overexpression of IL-7R in 
lung adenocarcinomas was associated with tumor budding, which in turn has been associated with unfavorable outcome. The tumor-immune interactions at the invading edge of tumors have been advocated as a driving force for their aggressiveness [41]. Functionally, IL7 has been shown to induce expression of VEGF-D and cyclin D in A549 cells and promote proliferation and lymphangiogenesis of lung cancer xenograft tumors [39]. Moreover, exogenous IL-7 has been demonstrated to reduce the percentage of apoptotic non-small cell lung cancer cells by up-regulating the expression of anti-apoptotic Bcl-2 and down-regulating the expression of pro-apoptotic Bax and p53 [42].

An interesting observation reported in this study is a significant variation in systemic IL-7 levels with respect to tumor location. Although collectively referred to as colorectal cancer, the disease is heterogeneous and argued to encompass three entities, i.e., cancers of the right or left colon and rectal cancer [43, 44]. In general, the right colon cancers are considered to be more aggressive, poorly differentiated, and associated with worse prognosis than the left colon cancers [45]. To address the differences between these two colon cancers Glebov at el. [44] identified over 1300 genes that were differently expressed by right and left colon. However, the issue of possible differences being reflected on systemic level has not been explored. We observed IL-7 to be significantly more elevated in right than left or rectal cancers, and the association was not mediated by differences in stage or metastatic lymph node distribution. Taking into account advocated link between IL-7 signaling and tumor aggressiveness and hence worse prognosis [11, 17], more pronounced IL-7 elevation in right colon cancers might contribute to unfavorable outcomes associated with that tumor location. Interestingly, a total lymphocyte count was significantly lower in patients with right compare to left colon location of the tumors (data not shown). If systemic IL-7 reflects its intestinal expression, one of possible explanations of differences in IL-7 with respect to tumor location might be related to differences in bacterial load and composition in these two locations. Yoshioka et al. [46] reported that bacterial products such as flagellin might suppress IL-7 production by intestinal epithelial cells.

The rationale behind IL-7-based therapies is its capability to selectively expand subpopulations of T lymphocytes. IL-7 preferentially increases number of recent thymic emigrants, naïve, and central memory $\mathrm{T}$ cells [7] as well as tumor-redirected cytotoxic $\mathrm{T}$ lymphocytes [12]. In turn, expansion of the regulatory $\mathrm{T}$ cells is negligible, rendering their percentage in total lymphocyte population relatively decreased [7]. Similarly, by up-regulating the count of other subsets, IL-7 relatively reduces percentage of the senescent $T$ cells [7]. Moreover, IL-7 protects T cells against tumor-induced senescence and thus abrogates their proangiogenic activity [47].
A number of researchers have recently reported successful application of IL-7. Wu et al. [48] used IL-7 to expand ex vivo $\mathrm{V} \delta 1 \mathrm{~T}$ cells isolated from peripheral blood, which then was successfully used to restrain the tumor growth and improved survival of mice with xenografted human colon carcinoma. Zhao et al. [49] reported IL-7 insertion to autologous tumor vaccine modified with a virus that enhanced cytotoxicity and production of IFN $\gamma$ of tumor-infiltrating CD8+ lymphocytes. CRC, like most other malignancies, is associated with unfavorable shift in immune responses toward Th2 and diminished total count of Th1 CD4+ cells, accompanied by reduced production of their prototypical cytokines IFN $\gamma$ and IL-12 (reviewed in [50]). Here, we examined the associations between IL-7 and other cytokines and growth factors in CRC. IL-7 was tightly and positively correlated with Th1 cytokines IFN $\gamma, T N F \alpha$ and IL-12(p70) and Th2 cytokine IL-10, of which TNF $\alpha$ and IL-10 remained significantly correlated following adjustment to other associations. IL-7 dependence on $\mathrm{TNF} \alpha$ is in agreement with stimulatory effect of TNF $\alpha$ on the expression of IL-7 [11]. We also observed IL-7 correlation with MCP-1 that might reflect cytokine effect on monocytes/macrophages. Zhang et al. [25] demonstrated that IL-7 induced macrophage activation and their homing into the gut, by inducing MCP-1 expression by intestinal epithelial cells.

In summary, our study is the first to demonstrate IL-7 elevation in CRC in association with lymph node involvement and tumor location. Both associations should be considered when designing IL-7-based immunotherapies. Further studies on IL-7 functionality in CRC are necessary.

Acknowledgements The research was supported by National Science Center, Grant\#DEC-2011/01/D/NZ5/02835 and is a part of the "WROVASC - Integrated Cardiovascular Centre" project co-financed by the European Regional Development Fund within the 2007-2013 Innovative Economy Operational Program realized in the Regional Specialist Hospital, Research and Development Centre in Wroclaw.

\section{Compliance with ethical standards}

Conflict of interest All authors hereby declare that they have no conflict of interest.

Open Access This article is distributed under the terms of the Creative Commons Attribution 4.0 International License (http://creativecommons.org/licenses/by/4.0/), which permits unrestricted use, distribution, and reproduction in any medium, provided you give appropriate credit to the original author(s) and the source, provide a link to the Creative Commons license, and indicate if changes were made.

\section{References}

1. Siegel RL, Sahar L, Portier KM, Ward EM, Jemal A (2015) Cancer death rates in US congressional districts. CA Cancer J Clin 65:339-344 
2. Chen W, Zheng R, Zeng H, Zhang S (2015) The updated incidences and mortalities of major cancers in China, 2011. Chin J Cancer 34:502-507

3. Armaghany T, Wilson JD, Chu Q, Mills G (2012) Genetic alterations in colorectal cancer. Gastrointest Cancer Res 5:19-27

4. Yamaji Y, Mitsushima T, Ikuma H, Watabe H, Okamoto M, Kawabe $T$ et al (2004) Incidence and recurrence rates of colorectal adenomas estimated by annually repeated colonoscopies on asymptomatic Japanese. Gut 53:568-572

5. Fleming M, Ravula S, Tatishchev SF, Wang HL (2012) Colorectal carcinoma: pathologic aspects. J Gastrointest Oncol 3:153-173

6. Koido S, Ohkusa T, Homma S, Namiki Y, Takakura K, Saito K et al (2013) Immunotherapy for colorectal cancer. World J Gastroenterol 19:8531-8542

7. Lundström W, Fewkes N, Mackall CL (2012) IL-7 in human health and disease. Semin Immunol 24:218-224

8. Fry TJ, Mackall CL (2002) Interleukin-7: from bench to clinic. Blood 99:3892-3904

9. Capitini CM, Chisti AA, Mackall CL (2009) Modulating T cell homeostasis with IL-7: preclinical and clinical studies. J Intern Med 266:141-153

10. Sportès C, Babb RR, Krumlauf MC, Hakim FT, Steinberg SM, Chow CK et al (2010) Phase I study of recombinant human interleukin-7 administration in subjects with refractory malignancy. Clin Cancer Res 16:727-735

11. Gao J, Zhao L, Wan YY, Zhu B (2015) Mechanism of action of IL-7 and its potential applications and limitations in cancer immunotherapy. Int J Mol Sci 16:10267-10280

12. Perna SK, Pagliara D, Mahendravada A, Liu H, Brenner MK, Savoldo B et al (2014) Interleukin-7 mediates selective expansion of tumor-redirected cytotoxic $\mathrm{T}$ lymphocytes without enhancement of regulatory Tcell inhibition. Clin Cancer Res 20:131-139

13. Rosenberg SA, Sportès C, Ahmadzadeh M, Fry TJ, Ngo LT, Schwarz SL et al (2006) IL-7 administration to humans leads to expansion of $\mathrm{CD} 8+$ and $\mathrm{CD} 4+$ cells but a relative decrease of CD4+ T-regulatory cells. J Immunother 29:313-319

14. Sportès C, Hakim FT, Memon SA, Zhang H, Chua KS, Brown MR et al (2008) Administration of rhIL-7 in humans increases in vivo TCR repertoire diversity by preferential expansion of naive T cell subsets. J Exp Med 205:1701-1714. doi:10.1084/ jem.20071681

15. Caprioli F, Marafini I, Facciotti F, Pallone F, Monteleone G (2013) Targeting T-cells in chronic inflammatory bowel diseases. J Clin Cell Immunol. doi:10.4172/2155-9899.1000155

16. Maeurer MJ, Walter W, Martin D, Zitvogel L, Elder E, Storkus W et al (1997) Interleukin-7 (IL-7) in colorectal cancer: IL-7 is produced by tissues from colorectal cancer and promotes preferential expansion of tumour infiltrating lymphocytes. Scand J Immunol 45:182-192

17. Al-Rawi MA, Mansel RE, Jiang WG (2003) Interleukin-7 (IL7) and IL-7 receptor (IL-7R) signalling complex in human solid tumours. Histol Histopathol 18:911-923

18. Mengus C, Le Magnen C, Trella E, Yousef K, Bubendorf L, Provenzano $\mathrm{M}$ et al (2011) Elevated levels of circulating IL-7 and IL-15 in patients with early stage prostate cancer. J Transl Med 9:162

19. Roato I, Caldo D, Godio L, D'Amico L, Giannoni P, Morello E et al (2010) Bone invading NSCLC cells produce IL-7: mice model and human histologic data. BMC Cancer 10:12

20. Berghella AM, Contasta I, Pellegrini P, Del Beato T, Adorno D (2002) Peripheral blood immunological parameters for use as markers of pre-invasive to invasive colorectal cancer. Cancer Biother Radiopharm 17:43-50
21. Crucitti A, Corbi M, Tomaiuolo PM, Fanali C, Mazzari A, Lucchetti D et al (2015) Laparoscopic surgery for colorectal cancer is not associated with an increase in the circulating levels of several inflammation-related factors. Cancer Biol Ther 16:671-677

22. Komura T, Sakai Y, Harada K, Kawaguchi K, Takabatake H, Kitagawa $\mathrm{H}$ et al (2015) Inflammatory features of pancreatic cancer highlighted by monocytes/macrophages and CD4+ T cells with clinical impact. Cancer Sci 106:672-686

23. Provatopoulou X, Georgiadou D, Sergentanis TN, Kalogera E, Spyridakis J, Gounaris A et al (2014) Interleukins as markers of inflammation in malignant and benign thyroid disease. Inflamm Res 63:667-674

24. Chen ZY, He WZ, Peng LX, Jia WH, Guo RP, Xia LP et al (2015) A prognostic classifier consisting of 17 circulating cytokines is a novel predictor of overall survival for metastatic colorectal cancer patients. Int J Cancer 136:584-592

25. Zhang W, Du JY, Yu Q, Jin JO (2015) Interleukin-7 produced by intestinal epithelial cells in response to Citrobacter rodentium infection plays a major role in innate immunity against this pathogen. Infect Immun 83:3213-3223

26. Ujiie H, Kadota K, Nitadori JI, Aerts JG, Woo KM, Sima CS et al (2015) The tumoral and stromal immune microenvironment in malignant pleural mesothelioma: a comprehensive analysis reveals prognostic immune markers. Oncoimmunology 4:e1009285

27. Henry CJ, Sedjo RL, Rozhok A, Salstrom J, Ahnen D, Levin TR et al (2015) Lack of significant association between serum inflammatory cytokine profiles and the presence of colorectal adenoma. BMC Cancer 15:123

28. Comstock SS, Xu D, Hortos K, Kovan B, McCaskey S, Pathak DR et al (2016) Association of serum cytokines with colorectal polyp number and type in adult males. Eur J Cancer Prev 25:173-181

29. Kim S, Keku TO, Martin C, Galanko J, Woosley JT, Schroeder JC et al (2008) Circulating levels of inflammatory cytokines and risk of colorectal adenomas. Cancer Res 68:323-328

30. Krzystek-Korpacka M, Diakowska D, Kapturkiewicz B, Bebenek M, Gamian A (2013) Profiles of circulating inflammatory cytokines in colorectal cancer (CRC), high cancer risk conditions, and health are distinct. Possible implications for CRC screening and surveillance. Cancer Lett 337:107-114

31. Krzystek-Korpacka M, Diakowska D, Neubauer K, Gamian A (2013) Circulating midkine in malignant and non-malignant colorectal diseases. Cytokine 64:158-164

32. Mattar MC, Lough D, Pishvaian MJ, Charabaty A (2011) Current management of inflammatory bowel disease and colorectal cancer. Gastrointest Cancer Res 4:53-61

33. Watanabe $M$, Watanabe $\mathrm{N}$, Iwao $\mathrm{Y}$, Ogata $\mathrm{H}$, Kanai $\mathrm{T}$, Ueno $\mathrm{Y}$ et al (1997) The serum factor from patients with ulcerative colitis that induces T-cell proliferation in the mouse thymus is interleukin-7. J Clin Immunol 17:282-292

34. Kader HA, Tchernev VT, Satyaraj E, Lejnine S, Kotler G, Kingsmore SF et al (2005) Protein microarray analysis of disease activity in pediatric inflammatory bowel disease demonstrates elevated serum PLGF, IL-7, TGF-beta1, and IL-12p40 levels in Crohn's disease and ulcerative colitis patients in remission versus active disease. Am J Gastroenterol 100:414-423

35. Korolkova OY, Myers JN, Pellom ST, Wang L, M'Koma AE (2015) Characterization of serum cytokine profile in predominantly colonic inflammatory bowel disease to delineate ulcerative and Crohn's colitides. Clin Med Insights Gastroenterol 8:29-44

36. Funderburg NT, Stubble Park SR, Sung HC, Hardy G, Clagett B, Ignatz-Hoover J et al (2013) Circulating CD4+ and CD8+T-cells are activated in inflammatory bowel disease and are associated with plasma markers of inflammation. Immunology 140:87-97 
37. Lee JC, Lyons PA, McKinney EF, Sowerby JM, Carr EJ, Bredin $F$ et al (2011) Gene expression profiling of CD8+ T-cells predicts prognosis in patients with Crohn disease and ulcerative colitis. J Clin Investig 121:4170-4179

38. Shive CL, Mudd JC, Funderburg NT, Sieg SF, Kyi B, Bazdar DA et al (2014) Inflammatory cytokines drive CD4+ T-cell cycling and impaired responsiveness to interleukin 7: implications for immune failure in HIV disease. J Infect Dis 210:619-629

39. Ming J, Zhang QF, Jiang YD, Jiang GC, Qiu XS (2015) Antilymphangiogenesis effects of a specific antiinterleukin 7 receptor antibody in lung cancer model in vivo. Mol Carcinog $54: 148-155$

40. Al-Rawi MA, Rmali K, Watkins G, Mansel RE, Jiang WG (2004) Aberrant expression of interleukin-7 (IL-7) and its signaling complex in human breast cancer. Eur J Cancer 40:494-502

41. Kadota K, Yeh YC, Villena-Vargas J, Cherkassky L, Drill EN, Sima CS et al (2015) Tumor budding correlates with the protumor immune microenvironment and is an independent prognostic factor for recurrence of stage I lung adenocarcinoma. Chest 148:711-721

42. Liu ZH, Wang MH, Ren HJ, Qu W, Sun LM, Zhang QF et al (2014) Interleukin 7 signaling prevents apoptosis by regulating bcl-2 and bax via the p53 pathway in human non-small cell lung cancer cells. Int J Clin Exp Pathol 7:870-881

43. Li F, Lai M (2009) Colorectal cancer, one entity or three. J Zhejiang Univ Sci B 10:219-229
44. Glebov OK, Rodriguez LM, Nakahara K, Jenkins J, Cliatt J, Humbyrd CJ et al (2003) Distinguishing right from left colon by the pattern of gene expression. Cancer Epidemiol Biomarkers Prev 12:755-762

45. Derwinger K, Gustawsson B (2011) Variations in demography and prognosis by colon cancer location. Anticancer Res $31: 2347-2350$

46. Yoshioka A, Okamoto R, Oshima S, Akiyama J, Tsuchiya K, Nakamura T et al (2008) Flagellin stimulation suppresses IL-7 secretion of intestinal epithelial cells. Cytokine 44:57-64

47. Ramello MC, Boari JT, Canale FP, Mena HA, Negrotto S, Gastman B et al (2014) Tumor-induced senescent $\mathrm{T}$ cells promote the secretion of pro-inflammatory cytokines and angiogenic factors by human monocytes/macrophages through a mechanism that involves Tim-3 and CD40L. Cell Death Dis 5:e1507

48. Wu D, Wu P, Wu X, Ye J, Wang Z, Zhao S et al (2015) Ex vivo expanded human circulating $\mathrm{V} \delta 1 \gamma \delta \mathrm{T}$ cells exhibit favorable therapeutic potential for colon cancer. Oncoimmunology 4:e992749

49. Zhao L, Mei Y, Sun Q, Guo L, Wu Y, Yu X et al (2014) Autologous tumor vaccine modified with recombinant new castle disease virus expressing IL-7 promotes antitumor immune response. J Immunol 193:735-745

50. Evans C, Dalgleish AG, Kumar D (2006) Immune suppression and colorectal cancer. Aliment Pharmacol Ther 24:1163-1177 